\title{
Specific features of the microbial resistance assessment in pig breeding enterprises
}

\author{
A. G. Isaeva ${ }^{1 \bowtie}$, A. S. Krivonogova ${ }^{2}$, I. M. Donnik ${ }^{1}$, K. V. Moiseeva ${ }^{1}$ \\ ${ }^{1}$ Ural State Agrarian University, Ekaterinburg, Russia \\ ${ }^{2}$ Ural Federal Agrarian Research Center of the Ural Branch of the Russian Academy of Sciences, Ekaterinburg, \\ Russia \\ ${ }^{凶}$ E-mail: isaeva.05@bk.ru
}

\begin{abstract}
The aim of this work was to study the opportunistic microbiocenosis of a pig-breeding enterprise and analyze its resistance to antimicrobial drugs (AMR-status). Methods. For two years on the pig farm there were taken samples of microflora from the mucous membranes and teats of pregnant and farrowed sows, from the mucous membranes and skin of piglets of weaning, rearing, fattening groups; from equipment, fencing, surfaces and inventory in different technological areas. The microorganism isolates isolated from the samples were determined for their sensitivity to antibiotics, which are most often used in veterinary practice and feeding in this region. Results. It was established that the surveyed enterprise has an unfavorable AMR-status. The nucleus of the opportunistic microbiocenosis was represented by S. aureus, Ent. faecium and P. aeruginosa, which accounted for about $44 \%$ of the isolated isolates, while the isolates of these microorganisms in $85 \%$ of cases had reduced sensitivity or resistance to one or more antibiotics. Multidrug resistance was found mainly in S. aureus isolates, which in $16 \%$ of cases were resistant to three antibiotics, and in $7 \%$ of cases - to six studied antibiotics of various classes. Most often, multidrug-resistant staphylococcus aureus was detected in sows and piglets in the weaning phase. Also, during the research, there was found a high resistance of opportunistic microflora isolates to tetracycline, which had been used in this farm for more than three years. More than $10 \%$ of tetracycline-resistant S. aureus and Ent. isolates were found on the skin and mucous membranes of fattening pigs. faecium, which indicated an increased risk of contamination of raw meat at the slaughter stage by microorganisms carrying genes for resistance to tetracyclines. The novelty of this work lies in obtaining relevant data on the AMR status of a pig-breeding facility and identifying the most unfavorable technological areas in terms of microbial resistance. The research is executed at the expense of a grant of the RSF (project No.18-16-00040).

Keywords: antimicrobial resistance, opportunistic microflora, antibiotic sensitivity, antibiotic resistance, pigs, AMR status, AR contamination, tetracycline, multi-resistance, pig breeding enterprise.
\end{abstract}

For citation: Isaeva A. G., Krivonogova A. S., Donnik I. M., Moiseeva K. V. Specific features of the microbial resistance assessment in pig breeding enterprises // Agrarian Bulletin of the Urals. 2020. No. 11 (202). Pp. 59-63. DOI: ...

Paper submitted: 04.08.2020.

\section{Introduction}

The high antimicrobial efficacy of antibiotics has led to their widespread use in all areas of medicine, veterinary medicine, and agriculture around the world. For decades, different types of antibiotics have been used not only for therapeutic but also for prophylactic purposes, especially in the industrial and food sectors. However, strains of resistant microorganisms were known even before the large-scale use of antibiotic therapy in the first half of the 20th century. High adaptive abilities of microorganisms contributed to the rapid activation of mechanisms of resistance to antimicrobial substances of various nature. The list of animals' and humans' infections, which are less and less responsive to antibiotic therapy, is growing every year. The World Health Organization has recognized that antibiotic resistance is one of the major threats to public health and food safety in the 21st century [1, p. 1235], [2]. Antibiotic-resistant infections are known to correlate with antibiotic consumption. [3 p. 226], [4, p. 227], [5, p. 2288]. Back in 1989, it was found that any form of antimicrobial action leads to an increase in the prevalence of antimicrobial resistance and multiple resistance of bacteria [6]. For a long time, antibiotics have been widely used in medicine and agriculture without control, assessment of feasibility, for prevention. This resulted in the spread of genes for resistance to antimicrobial drugs, including antibiotics, antituberculosis, antiviral, antiparasitic and antifungal drugs, in microbiocenoses [2], [7]. The antibiotic resistance gene $(A R G)$ is now considered a new contaminant. Studies have shown that livestock enterprises are one of the main reservoirs of AR genes [8, p. 820], [1, p. 1242]. Microbiome components carrying antibiotic resistance genes enter the environment along with animal excrement, accelerating the spread of ARGs in the environment; in addition, at some technological stages of production and processing of animal raw materials, they can enter the raw materials, enter the food chain and be transmitted to humans. Thus, the contamination of biocenoses and food chains with microorganisms and AR genes presents a new threat to public health. [1, 1242], [9, p. 1], [10, p. 48]. The global action plan to combat antimicrobial resistance, adopted 
by WHO in 2015, declared monitoring of microbial resistance, control of antibiotics in veterinary medicine and agriculture, scientific search for AMR containment mechanisms [11], [12]. Currently, the study of the mechanisms of bacteria interaction with antibiotics using molecular biological analysis of the phenomena of resistance has made it possible to increase the effectiveness of antibiotic therapy without increasing the risk of spreading antimicrobial resistance (AMR) [13, p. 91]. There are technologies and vaccine prevention of a number of bacterial infections, the treatment of which with antibiotics is difficult due to the high level of resistance - for example, immunization against pneumococcus and prevention of pneumonia caused by it in humans [14, p. 96].

However, in the field of livestock, the fight against AMR lags behind that in health care both in quality and in terms of coverage. Thus, the introduction of microbial resistance passports at livestock facilities in the Russian Federation is still experimental, isolated. It is known that the level of contamination of livestock systems with AR-agents (including genes and bacteria themselves) is constantly growing all over the world $[15$, p. 1251$]$. At the same time, according to the available literature data, countries with low and middle income, including the Russian Federation, do not adequately control and do not regulate the use of antibiotics, as a result of which the situation with the growth of antimicrobial resistance (AMR) in them remains unfavorable $[15$, p. 1], [14, p. 95]. The methods of leveling technological errors in preventive antibiotic therapy widespread in animal husbandry, the use of antibiotic-containing feed additives in pig breeding to increase the adaptive capabilities of livestock under constant stress, inevitably leads to a deterioration in the AMR status of the enterprise. However, prophylactic antibiotics can make any antibiotic therapy ineffective for this population, therefore it is prohibited in developed countries. [16, p. 52]. Resistant staphylococci, enterococci, Pseudomonas aeruginosa, Proteus, Escherichia coli, Klebsiella and ARG are considered to be unwanted microbiome contaminants in pig production. [17, p. 37] [10, p. 48], $[18$, p. 277]. Multi-resistant isolates of these microorganisms are becoming a new problem for pig breeding, since in addition to the ineffectiveness of antibiotic therapy, they also reduce the quality of meat raw materials and products. Meat contaminated with antibiotic resistance isolates or resistance determinants becomes a factor in the transmission of AMR to the consumer through the food chain. [18, p. 280]. [15, p. 1251]. In addition, the release of resistant microorganisms and ARG into the environment with waste from pig farms contributes to the contamination of soil, water bodies, plants, wild animals, and, in general, leads to a deterioration of the AMR status of the area. $[19$, p. 83.] [20, p. 430], [21, p. 60]. [5, p. 2290]. In this regard, it seems relevant to study the opportunistic microbiocenosis and its AMR status at pig breeding facilities.

The aim of the research was to study the characteristics of the opportunistic microbiocenosis of a pig-breeding facility and to assess the level of spread of antimicrobial resistance (AMR). To achieve the goal, the following tasks were implemented: was carried out a structural analysis of opportunistic microbiocenosis at pig breeding facilities; was performed the analysis of the sensitivity of isolates to antibiotics.

\section{Methods}

The research was carried out in 2018-2019 at a pig breeding enterprise in the Sverdlovsk region. There were taken samples of air, feed and premixes, bedding, water for drinking, washings were made from mucous membranes and teats of pregnant and farrowed sows, washings from mucous membranes and skin of piglets of weaning, rearing, fattening groups; flushes from equipment, fencing, surfaces and inventory in different areas and in different points of technological rooms. The collected samples were analyzed according to standard microbiological methods. Sowing on nutrient media, cultivation, isolation of a pure line, identification of isolates, and pathogenicity were determined [22]. We studied the sensitivity of the isolated isolates to the classes of antibiotics most often used in veterinary practice and feeding: ampicillin, amoxicillin (semisynthetic penicillins), meropenem (carbapenems), cefazolin (cephalosporin I generation), tetracycin (fluoroquinolones, III generation), van (glycopeptides), rifampicin (ansamycins), azithromycin (macrolides). The analysis of antibiotic susceptibility was performed by the disk diffusion method. The data were statistically processed using the STATISTICA 10 program.

\section{Frequency distribution of isolates on the surveyed pig farm in 2019}

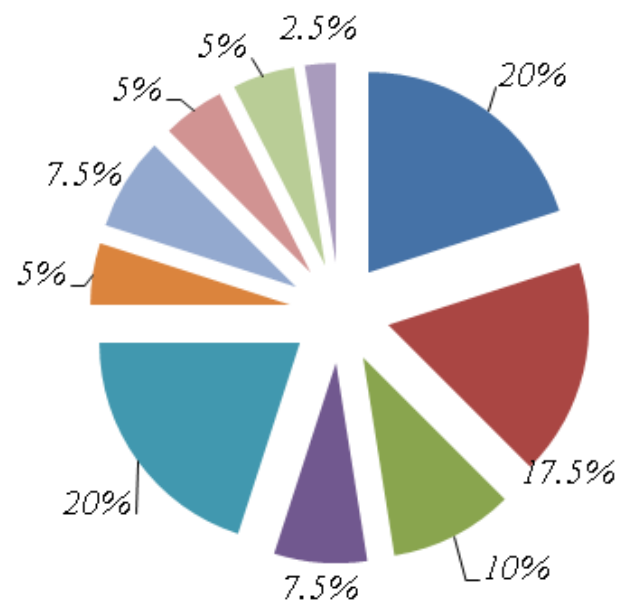

\author{
Enterococcusfaecium \\ - Staphylococcus aureus \\ - Pseudomonas aeruginosa \\ -Aspergillus spp. \\ - Candida albicans \\ Enterobacter spp. \\ Echerichiacoli \\ Proteus spp. \\ Penicillium \\ - Staphylococcusepidermidis
}

Fig. 1. Frequency distribution of isolates that make up the core of the opportunistic microbiocenosis on the surveyed pig farm in 2019 
Antibiotic sensitivity of isolates found in the sows area (2019)
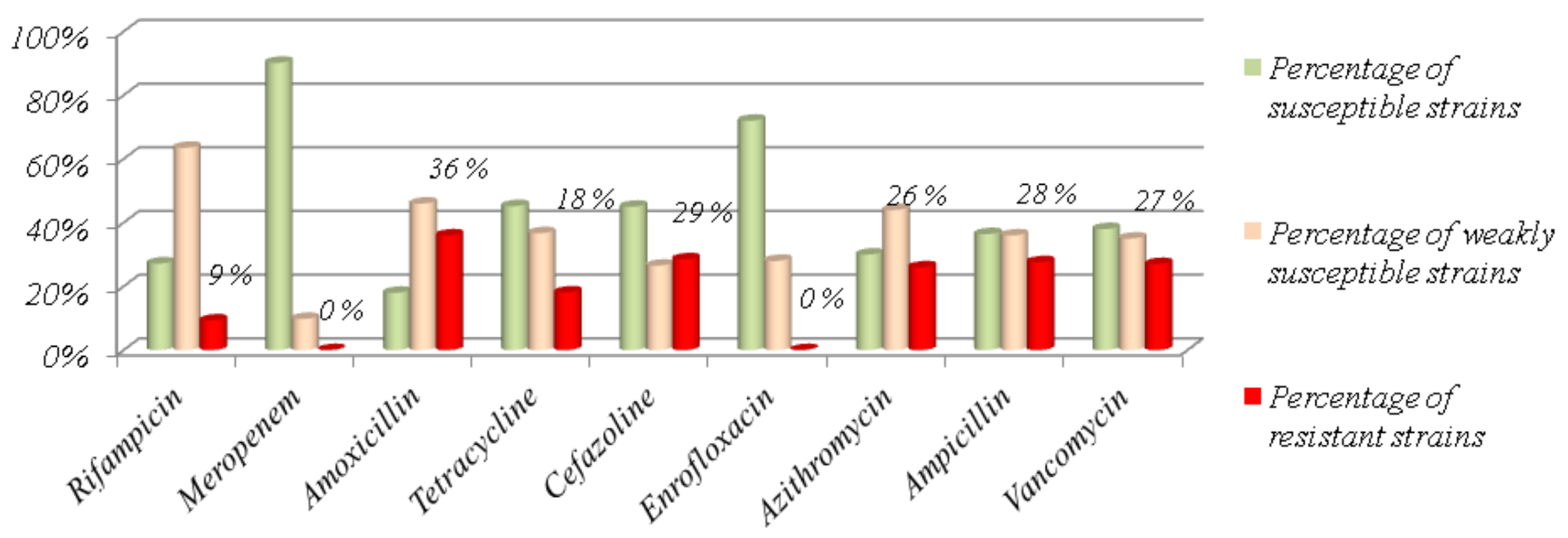

Fig. 2. Antibiotic sensitivity of opportunistic pathogenic microorganism isolates found in the sows area

Results

Microbiological studies carried out at a pig-breeding enterprise showed that the species composition of the opportunistic part of the microbiota was relatively the same for two years. Most often, isolates of enterococci, Staphylococcus aureus, Pseudomonas aeruginosa were found in the samples. The average distribution of isolates over two years of observation by frequency of occurrence in samples was as follows: Ent. faecium $(19.2 \%)$, C. albicans (14.3\%), S. aureus (13.1\%), P. aeruginosa (12.3\%), Aspergillus spp. (9.2\%), Enterobacter (6.8\%), Proteus spp. (6.2\%), E. coli (5.6\%), S. saprophyticus (3.7\%), S. epidermidis (2.3\%), Penicillium spp. (1.8\%). Isolates Ent. faecalis, Ent. durans, Klebsiella spp., Fusaium spp., Mucor spp., were found less often - in total, their share did not exceed $5.5 \%$ in this distribution.

Significant two-year dynamics for 2018-2019 in the ratio of isolates in the nucleus of the opportunistic microbiocenosis on the surveyed pig farm was revealed for C. albicans (an increase of $11.3 \%$ ), S. aureus (an increase of $3.5 \%$ ), E. coli (an increase of 2 times). The share of Ent has increased. faecium, S. epidermidis, decreased proportion of Penicillium spp., Aspergillus spp. (fig. 1). In general, the constancy of the presence of the main isolates of opportunistic microflora was noted in samples from all objects - from the mucous membranes and skin of sows, piglets, from fences, equipment, implements, in samples, litter, feces, feed, water and air. The ratio of microorganisms was different from the typical farm only in the case of samples of manure and litter. These samples were dominated by isolates of enterococci (in $100 \%$ of the samples, isolates of Ent. Faecium were found), Proteus; Pseudomonas aeruginosa isolates were relatively rare (in 5-8\% of samples).

We established a high level of contamination of the number of pregnant sows with C. albicans, S. aureus and P. aeruginosa. Isolates of these microorganisms were found in $50-70 \%$ of swabs from the mucous membranes of the mouth, nose and vagina. In the inventory (scrapers, shovels) in all surveyed areas, isolates of Enterococcus, Staphylococcus aureus, Pseudomonas aeruginosa, Proteus (the ratio of $\mathrm{P}$. vulgaris and $\mathrm{P}$. mirabilis averaged 7:1), Penicillium, Aspergillus were found. The total microbial count in air samples averaged $2900-3200 \mathrm{CFU} / \mathrm{m}^{3}$, which indicated a high microbial load. From air samples, mainly mold fungi, enterococcus and Staphylococcus aureus were sown.

We investigated the AMR-status of the detected isolates. The sensitivity of bacteria to antibiotics used in this farm for at least three years (tetracycline, cefazolin, ampicillin), as well as to antibiotics most often used in pig farms in the Ural region (amoxicillin, meropenem, enrofloxacin, vancomycin, rifampicin, azithromycin). All these drugs, with the exception of enrofloxacin, are used not only in veterinary but also in medical practice for the treatment of infectious, surgical and purulentseptic pathology in humans. Therefore, the contamination of raw materials, products and the environment with bacteria and genes for resistance to these antibiotics can pose a risk for both farm employees and consumers of its products, and timely detection of resistant isolates at a pig breeding enterprise is important not only for animals.

When processing the test results, only those microorganisms were taken into account that are included in the spectrum of action of a particular antibiotic, are targets for it, and in relation to which the mechanism of bactericidal or bacteriostatic action of this drug is realized in a minimum inhibitory concentration. Microorganisms with natural resistance to a particular class of antibiotics were not considered. Cases of dosedependent effects, native moderate and weak sensitivity of the microorganism to the antibiotic were not taken into account.

The greatest number of episodes of resistance was detected at the processing area of sows. The samples contained isolates resistant to rifampicin, ampicillin and amoxicillin, vancomycin, cefazolin and tetracycline. At the same time, the highest resistance was recorded to amoxicillin, which was used in this farm for less than two years (about $36 \%$ of isolated microorganisms were immune). $28 \%$ of isolated isolates were resistant to ampicillin (fig. 2). This fact indicates the initially high status of antimicrobial resistance for ampicillin and its protected variant - amoxicillin - on the surveyed pig farm, which may be associated with a long period of amoxicillin use in previous years or with the introduction of resistant microorganisms and AMR genes into the farm from the outside. 


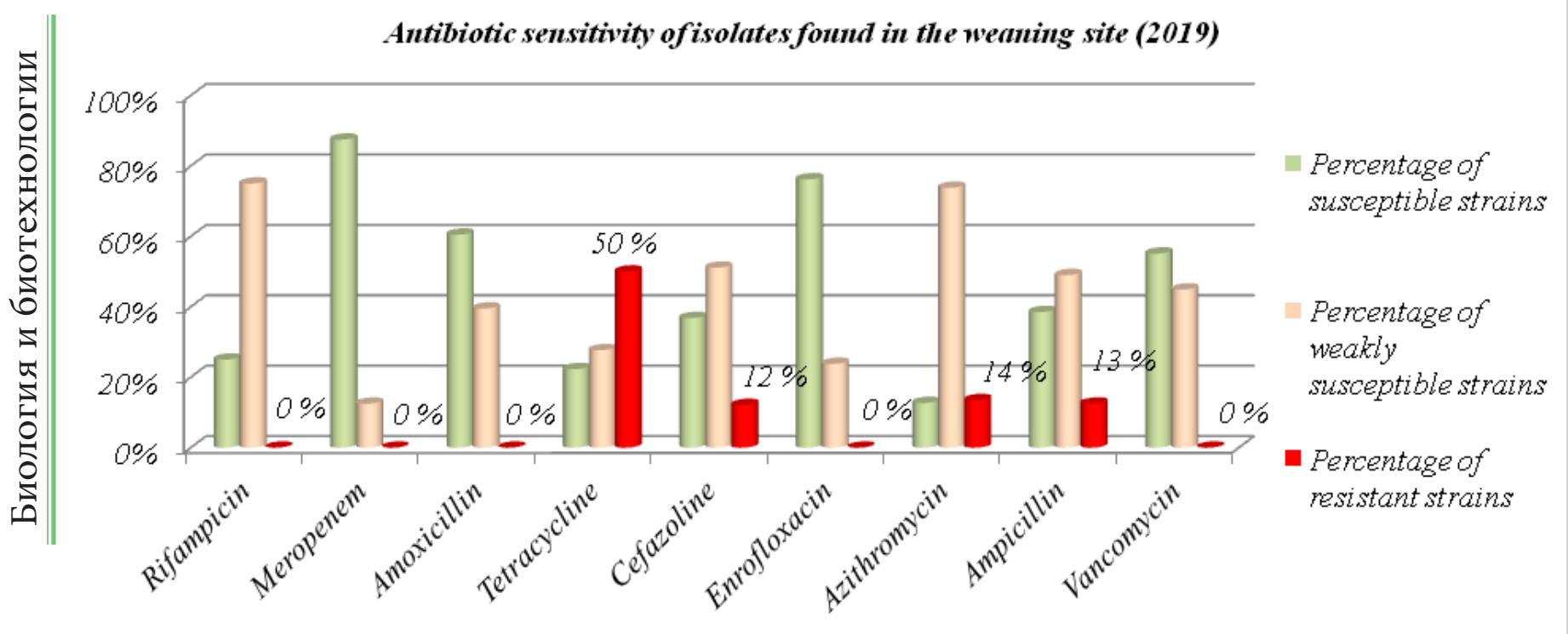

Fig. 3. Antibiotic sensitivity of opportunistic pathogenic microorganisms isolates found at the weaning site

At the technological site, where piglets are kept in the weaning phase, the AMR status of the microbiocenosis looked different: the most unfavorable antibiotic was tetracycline, half of all detected isolates were resistant to it (fig. 3).

In the growing area, tetracycline-resistant isolates remained, but the frequency of their detection decreased by half (to $25 \%$ ). Resistance to ampicillin (12\%) and rifampicin (12\%) was also found. In the feeding area, $13 \%$ of tetracycline-resistant isolates were found. Thus, tetracycline resistance in this pig farm has been identified in all key processing areas. The retention of resistant isolates during the feeding phase increases the risk of AR genes and bacterial contamination of raw meat during slaughter and cutting.

In general, when analyzing the AMR status of the opportunistic microbiocenosis of a pig farm, the greatest number of episodes of resistance was found in S. aureus isolates. Reduced sensitivity to one antibiotic was detected in more than $85 \%$ of detected staphylococci. More than $64 \%$ of all isolates of this microorganism showed resistance to antibiotics of at least one class, in $16 \%$ of cases they showed multiresistance to 3 classes of ABP, in $7 \%$ of cases - multiresistance to six classes of ABP: to semisynthetic penicillins, carbapenems, tetracyclines, fluoroquinolones and glycopeptides). Such multi-resistant S. aureus isolates. found on the mucous membranes of the vagina, oral cavity in sows and piglets from weaning and growing groups; from samples of litter and swabs from inventory in the premises where these animals were kept. Sensitivity analysis of $\mathrm{P}$. aeruginosa and Ent isolates. faecium showed that about $38 \%$ of all isolated Ent. faecium were insensitive to at least one drug (most often to tetracycline, ampicillin, vancomycin); more than half of P. aeruginosa isolates were resistant or had extremely low sensitivity to macrolides and fluoroquinolones.

A general analysis of cases of multiple resistance over a two-year period showed the following: the total number of cases of resistance to two classes of ABD was $9.2 \%$ of all isolated strains; to 3 classes of antibiotics $-5.5 \%$, and to 5-6 classes $-2.7 \%$. The growth in 2019 compared to 2018 was $0.7 \%, 1.0 \%$ and $0.5 \%$, respectively. No super-resistant strains resistant to all $8 \mathrm{ABP}$ classes that were in the work were not found.

\section{Discussion and Conclusion}

The studies carried out have shown that there is a pool of antibiotic-resistant microorganisms at the pig breeding enterprise, which make up a relatively stable core of opportunistic microbiocenosis. Fluctuations in the proportions of different microorganisms in the structure of the microbiocenosis during two years were quite noticeable, but the proportion of the main representatives $-\mathrm{S}$. aureus, Ent. faecium and P. aeruginosa remained the largest, accounting for a total of $44 \%$ of the isolated isolates. At the same time, the detected isolates of these microorganisms were characterized by a high level of resistance to antibiotics. More than $85 \%$ of the isolates had either reduced sensitivity to the studied antibiotics or were resistant to one of them. The greatest number of cases of multiple resistance was found in $\mathrm{S}$. aureus isolates, which in $16 \%$ of cases were resistant to three and in $7 \%$ of cases to six antibiotics of various classes. Especially significant is the fact that multidrug-resistant isolates of Staphylococcus aureus have been detected in sows and weaning sites. The highest level of total resistance was found on the site of sows, which, most likely, is associated with the duration of keeping pigs for reproductive purposes. At this site, there was a risk of contamination of newborn piglets with staphylococcus strains with a high level of antibiotic resistance. It was found that tetracycline, which had been used in this farm for a long time, had lost its effectiveness - half of the isolates isolated at the technological site of weaning were resistant to it. More than $10 \%$ of tetracycline-resistant isolates, mainly S. aureus and Ent. faecium. This fact indicates a high risk of contamination of meat raw materials at the slaughter stage by microorganisms carrying genes for resistance to tetracyclines.

The results of our studies indicate that the AMR status of the surveyed enterprise is not well and about the need to take measures to contain antimicrobial resistance, eliminate resistant strains and protect raw meat from contamination with AMR agents.

\section{Acknowledgements}

The research is executed at the expense of a grant of the RSF (project No.18-16-00040). 


\section{References}

1. Shi X., Wang S. Antibiotic resistance in environment of animal farms // Chinese journal of biotechnology. 2018. V. 34. Pp. 1234-1245.

2. Global Framework for Development \& Stewardship to Combat Antimicrobial Resistance. Draft Roadmap. WHO/EMP/ IAU/ 2017.08 (revised 19 October 2017) [e-resource]. URL: https://www.who.int/antimicrobial-resistance/global-action-plan/ UpdatedRoadmap-Global-Framework-for-Development-Stewardship-to-combatAMR_2017_11_03.pdf?ua=1 (appeal date: 25.09.2019)

3. Sandru F., Popa A., Dumitrascu M., Petca A., Petca R. C., Draghici C., Stănescu A. New perspectives on antibiotic resistance // Romanian Medical Journal. 2019. T. 66. Pp. 226-228.

4. Zaman S. B. Hussain M., Nye R., Mehta V., Mamun T., Hossain N. A Review on Antibiotic Resistance: Alarm Bells are Ringing // Cureus. 2017. No. 9 (6). e. 1403.

5. Gellin G., Gellin L., Dawson K., Aaron D. Antibiotic resistance of Gram-negative enteric bacteria from pigs in three herds with different histories of antibiotic exposure // Applied and environmental microbiology. 1989. T. 55. Pp. $2287-2292$.

6. Akhtar S., Choudhary N., Rasheed M. Antibiotic Resistance: A Global Phenomenon // Indian Journal Of Applied Research. 2019. Vol. 9. Iss. 2. Pp. 52-58.

7. Order of the Government of the Russian Federation of September 25, 2017 No. 2045-r "On the Strategy for preventing the spread of antimicrobial resistance in the Russian Federation for the period up to 2030. Strategy for preventing the spread of antimicrobial resistance in the Russian Federation for the period until 2030". Approved by the order of the Government of the Russian Federation of September 25, 2017 No. 2045-r [e-resource]. URL: https:/www.garant.ru/products/ipo/prime/ doc/71677266/\#review (appeal date: 17.08.2019).

8. Hasan Abusaiba T. AL-Harmoosh R. Mechanisms of Antibiotics Resistance in Bacteria // Systematic Reviews in Pharmacy. 2020. V. 11.Pp. 817-823.

9. Ventola L. C. The antibiotic resistance crisis. Part I: Causes and Threats // Pharmacy and Therapeutics. 2015. No. 40. Pp. 277-283.

10. Lartseva L. V., Obukhova O. V., Barmin A. N. Ekologicheskaya i biologicheskaya opasnost' rezistentnosti uslovno-patogennoj mikroflory $\mathrm{k}$ antibiotikam (obzor) [Environmental and biological danger of resistance of opportunistic microflora to antibiotics (review)] // Russian Journal of Applied Ecologyii. 2015. No. 4 (4). Pp. 47-52. (In Russian.)

11. Global action plan on antimicrobial resistance. Sixty-eighth World Health Assembly. WHA68.7 (Agenda item 15.126 May 2015) [e-resource]. URL: https://apps.who.int/gb/ebwha/pdf files/WHA68/A68 R7-en.pdf?ua=1 (appeal date: 17.08.2019).

12. Terrestrial Animal Health Code, Terrestrial Animal Health Code. Chapter 6.10. Responsible and prudent use of antimicrobial agents in veterinary medicine [e-resource]. URL: https:/www.oie.int/fileadmin/Home/eng/Health_standards/tahc/current/ chapitre antibio use.pdf (appeal date: 17.08.2019).

13. Ete $\bar{b}$ E. Arikekpar I. Antibiotics: Classification and mechanisms of action with emphasis on molecular perspectives // International Journal of Applied Microbiology and Biotechnology Research. 2016. Vol. 4. Pp. 90-101.

14. Lewnard J. A. Lo N. C., Arinaminpathy N. Childhood vaccines and antibiotic use in low- and middle-income countries // Nature. 2020. T. 581. Pp. 94-99.

15. Moore C. Changes in antibiotic resistance in animals // Science. 2019. Vol. 365. Pp. 1251-1252.

16. Mathew A. Achieving sustainable production of pig meat. Vol. 1. London: Burleigh Dodds Science Publishing, 2018.290 p.

17. Khan M. F. Brief History of Staphylococcus aureus: A Focus to Antibiotic Resistance // EC Microbiology. 2017. No. 5. Pp. 36-39.

18. Fard R. Heuzenroeder M., Barton M. Antimicrobial and heavy metal resistance in commensal enterococci isolated from pigs // Veterinary microbiology. 2010. No. 148. Pp. 276-282. DOI: 10.1016/j.vetmic.2010.09.002.

19. Barton M. D. Antibiotic use in animal feed and its impact on human health // Nutrition Research Reviews. 2000. No. 13. Pp. 279-299.

20. Krzeminski P., Popowska M. Treatment Technologies for Removal of Antibiotics, Antibiotic Resistance Bacteria and Antibiotic-Resistant Genes // Antibiotics and Antimicrobial Resistance Genes. Emerging Contaminants and Associated Treatment Technologies. Springer, Cham. 2020. Pp. 415-434.

21. Ebner P., Yingying H. Dealing with the challenge of antibiotic resistance in pig production // Achieving sustainable production of pig meat. Volume 1: Safety, quality and sustainability. Burleigh Dodds Science Publishing. 2018. Pp. 51-66.

22. Klinicheskie rekomendacii. Opredelenie chuvstvitel'nosti mikroorganizmov k antimikrobnym preparatam [Clinical recommendations. Determination of the susceptibility of microorganisms to antimicrobials] [e-resource]. 2015. URL: http://www. antibiotic.ru/minzdrav/files/docs/clrec-dsma2018.pdf (appeal date: 18.08.2019). (In Russian.)

\section{Authors'information:}

Albina G. Isaeva ${ }^{1}$, doctor of biological sciences, associate professor of the department of infectious and non-infectious pathology, ORCID 0000-0001-8395-124, AuthorID 665717; +7982 872-89-10,isaeva.05@bk.ru

Anna S. Krivonogova ${ }^{2}$, doctor of biological sciences, associate professor, leading researcher, ORCID 0000-0003-1918-3030, AuthorID 683239; +7982 651-29-34

Irina M. Donnik ${ }^{1}$, doctor of biological sciences, professor, academician of the Russian Academy of Sciences, head of the department of infectious and non-infectious pathology, ORCID 0000-0001-8349-3004, AuthorID 313786

Ksenia V. Moiseeva ${ }^{1}$, assistant of the department of infectious and non-infectious pathology, ORCID 0000-0002-9858-1880, AuthorID 779572; +7 953 047-58-87

${ }^{1}$ Ural State Agrarian University, Ekaterinburg, Russia

${ }^{2}$ Ural Federal Agrarian Research Center of the Ural Branch of the Russian Academy of Sciences, Ekaterinburg, Russia 